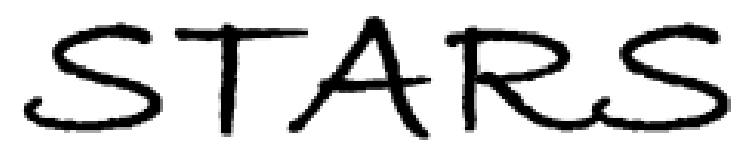

University of Central Florida

STARS

Faculty Bibliography 2000s

Faculty Bibliography

$1-1-2006$

\title{
Spectroscopic rotational velocities of brown dwarfs
}

M. R. Zapatero Osorio

E. L. Martin

University of Central Florida

H. Bouy

R. Tata

University of Central Florida

R. Deshpande

University of Central Florida

See next page for additional authors

Find similar works at: https://stars.library.ucf.edu/facultybib2000

University of Central Florida Libraries http://library.ucf.edu

This Article is brought to you for free and open access by the Faculty Bibliography at STARS. It has been accepted for inclusion in Faculty Bibliography 2000 s by an authorized administrator of STARS. For more information, please contactSTARS@ucf.edu.

\section{Recommended Citation}

Osorio, M. R. Zapatero; Martin, E. L.; Bouy, H.; Tata, R.; Deshpande, R.; and Wainscoat, R. J., "Spectroscopic rotational velocities of brown dwarfs" (2006). Faculty Bibliography 2000s. 7869.

https://stars.library.ucf.edu/facultybib2000/7869

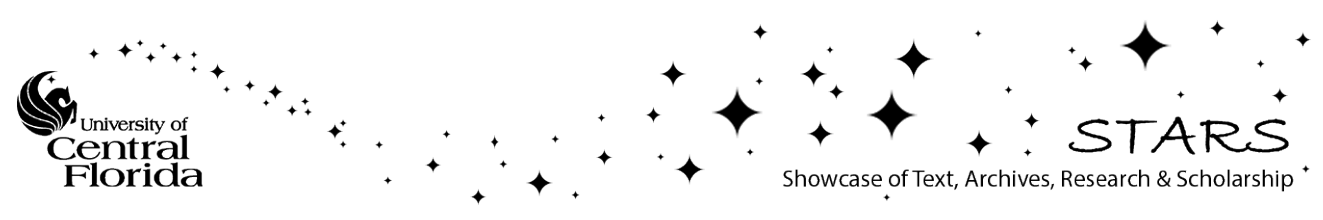




\section{Authors}

M. R. Zapatero Osorio, E. L. Martin, H. Bouy, R. Tata, R. Deshpande, and R. J. Wainscoat 


\title{
SPECTROSCOPIC ROTATIONAL VELOCITIES OF BROWN DWARFS
}

\author{
M. R. ZAPATERo OSORIO ${ }^{1}$ \\ LAEFF-INTA, P.O. Box 50727, E-28080 Madrid, Spain; mosorio@laeff.inta.es \\ E. L. Martín ${ }^{2}$ and H. BouY \\ Instituto de Astrofisica de Canarias (IAC), Vía Láctea s/n, E-38205 La Laguna, Tenerife, Spain; ege@iac.es, bouy@iac.es \\ R. TAta and R. Deshrande \\ University of Central Florida, Department of Physics, P.O. Box 162385, Orlando, FL 32816-2385; tata@physics.ucf.edu, rohit@physics.ucf.edu \\ AND \\ R. J. WAINSCOAT \\ Institute for Astronomy, 2680 Woodlawn Drive, Honolulu, HI 96822; rjw@ifa.hawaii.edu \\ Received 2006 March 7; accepted 2006 April 28
}

\begin{abstract}
We have obtained projected rotation velocities $\left(v_{\text {rot }} \sin i\right)$ of a sample of 19 ultracool dwarfs with spectral types in the interval M6.5-T8 using high-resolution, near-infrared spectra obtained with NIRSPEC and the Keck II telescope. Among our targets there are two young brown dwarfs, two likely field stars, and 15 likely brown dwarfs (30-72 $\left.M_{\mathrm{Jup}}\right)$ in the solar neighborhood. Our results indicate that the T-type dwarfs are fast rotators in marked contrast to M-type stars. We have derived $v_{\text {rot }} \sin i$ velocities between $\leq 15$ and $40 \mathrm{~km} \mathrm{~s}^{-1}$ for them and have found no clear evidence for $\mathrm{T}$ dwarfs rotating strongly faster than $\mathrm{L}$ dwarfs. However, there is a hint for an increasing lower envelope on moving from mid-M to L spectral types in the $v_{\text {rot }} \sin i$-spectral-type diagram that was previously reported in the literature; our $v_{\text {rot }} \sin i$ results extend it to even cooler types. Assuming that field brown dwarfs have a size of $0.08-0.1 R_{\odot}$, we can place an upper limit of $12.5 \mathrm{hr}$ on the equatorial rotation period of T-type brown dwarfs. In addition, we have compared our $v_{\text {rot }} \sin i$ measurements to spectroscopic rotational velocities of very young brown dwarfs of similar mass available in the literature. The comparison, although model dependent, suggests that brown dwarfs lose some angular momentum during their contraction; however, their spin-down time seems to be significantly longer than that of solar-type to early $\mathrm{M}$ stars.

Subject headings: stars: individual (DENIS-P J0255.0-4700, GL 570D, LP 944-20, [Pleiades] PP1 1, SDSS J053951.99-005902.0, SDSS J134646.45-003150.4, SDSS J162414.37+002915.6, SDSSp J125453.90-012247.4, 2MASS J00361617+1821104, 2MASS J03341218-4953322, 2MASS J04151954-0935066, 2MASS J05591914-1404488, 2MASS J12171110-0311131, 2MASS J15031961+2525196, 2MASS J15530228+1532369AB, 2MASS J16322911+1904407, 2MASS J17281150+3948593AB, 2MASS J22244381-0158521, vB 10) -

stars: low-mass, brown dwarfs — stars: rotation
\end{abstract}

\section{INTRODUCTION}

Rotation is a key parameter fundamental in examining stellar activity and angular momentum evolution. The rotation of stars has been studied for nearly all spectral types and for a wide range of ages. These studies have contributed to our knowledge of the stellar angular momentum history. In particular, field early $\mathrm{M}$ dwarfs are among the slowest rotating stars on the main sequence, with typical projected rotational velocities $\left(v_{\text {rot }} \sin i\right)$ below $5 \mathrm{~km} \mathrm{~s}^{-1}$ (e.g., Marcy \& Chen 1992; Delfosse et al. 1998). It has been suggested that many of these stars lose about $98 \%$ of their angular momentum during their lifetimes via magnetic activity, winds, and mass loss. However, very little is known about the rotation rate and angular momentum evolution of brown dwarfs.

The state-of-the-art evolutionary models by Baraffe et al. (2003) and Burrows et al. (1997) predict that a star at the substellar mass limit $\left(0.072 M_{\odot}\right.$, or $\left.\sim 72 M_{\text {Jup }}\right)$ reaches the hydrostatic equilibrium

\footnotetext{
1 Also at IAC, Vía Láctea s/n, E-38205 La Laguna, Tenerife, Spain.

2 Also at University of Central Florida, Department of Physics, P.O. Box 162385, Orlando, FL 32816-2385.
}

at an effective temperature of about $1800 \mathrm{~K}$. However, T-type dwarfs (for the spectral definition see Burgasser et al. [2002a] and Geballe et al. [2002]) are characterized by surface temperatures cooler than $\sim 1300 \mathrm{~K}$ (Vrba et al. 2004; Nakajima et al. 2004). Hence, T-type dwarfs are all believed to be brown dwarfs because they have cooled down below even the minimum atmospheric temperature of the smallest stars. On the basis of theoretical predictions and very recent temperature calibrations (e.g., Dahn et al. 2002), the star-brown dwarf frontier happens at spectral type L3-L4 at ages of a few gigayears. Field objects with later types are probably substellar. Various efforts have been made so far to derive the rotational velocity of L-type dwarfs (e.g., Basri et al. 2000; Reid et al. 2002; Mohanty \& Basri 2003; Bailer-Jones 2004). In contrast to early and mid-M stars, L dwarfs appear to be relatively fast rotators with $v_{\text {rot }} \sin i$ values in the range $10-60 \mathrm{~km} \mathrm{~s}^{-1}$. To the best of our knowledge, only the binary T1/T6 $\epsilon$ Indi Bab has a rotation velocity measurement available in the literature, $v_{\text {rot }} \sin i=28 \mathrm{~km} \mathrm{~s}^{-1}$ (determined for the unresolved system; Smith et al. 2003).

This paper presents high-resolution, near-infrared spectroscopic observations of 19 bright, ultracool dwarfs with spectral types in the interval M6.5-T8. Our analysis is intended to, first, 
TABLE 1

KeCK/NIRSPEC ObSERVING LoG

\begin{tabular}{|c|c|c|c|c|c|c|}
\hline Object & Observation Date & Slit Name & $\begin{array}{l}\text { Echelle } \\
\text { (deg) }\end{array}$ & $\begin{array}{c}\text { Exposure Time } \\
\text { (s) }\end{array}$ & Air Mass & Reference \\
\hline \multirow[t]{2}{*}{ 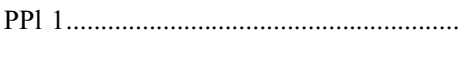 } & 2005 Oct 26 & $0.432 \times 12$ & 63.00 & $3 \times 480$ & 1.01 & \multirow[t]{2}{*}{1} \\
\hline & 2005 Oct 28 & $0.432 \times 12$ & 63.00 & $3 \times 420$ & 1.02 & \\
\hline \multirow[t]{2}{*}{ 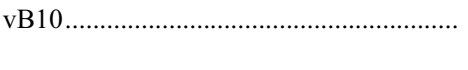 } & 2001 Jun 15 & $0.576 \times 12$ & 63.00 & $2 \times 300$ & 1.14 & \multirow[t]{2}{*}{2} \\
\hline & 2001 Nov 02 & $0.432 \times 12$ & 63.29 & $8 \times 120$ & 1.40 & \\
\hline \multirow[t]{2}{*}{ DENIS-P J033411.39-495333.6 ${ }^{\mathrm{a}} \ldots \ldots \ldots \ldots$} & 2005 Oct 26 & $0.432 \times 12$ & 63.00 & $6 \times 300$ & 2.86 & \multirow[t]{2}{*}{3} \\
\hline & 2005 Oct 28 & $0.432 \times 12$ & 63.00 & $3 \times 300$ & 2.86 & \\
\hline \multirow[t]{5}{*}{ 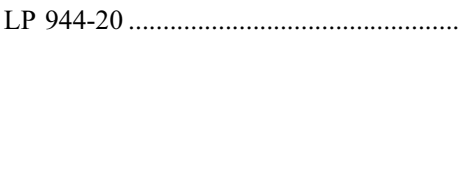 } & 2000 Dec 12 & $0.432 \times 12$ & 63.00 & $3 \times 200$ & 1.89 & \multirow[t]{5}{*}{4} \\
\hline & 2005 Oct 26 & $0.432 \times 12$ & 63.00 & $6 \times 300$ & 1.86 & \\
\hline & 2005 Oct 27 & $0.432 \times 12$ & 63.00 & $6 \times 300$ & 1.75 & \\
\hline & 2005 Oct 28 & $0.432 \times 12$ & 63.00 & $6 \times 300$ & 1.76 & \\
\hline & 2005 Oct $29^{b}$ & $0.432 \times 12$ & 63.00 & $1 \times 300$ & 1.80 & \\
\hline \multirow[t]{2}{*}{ 2MASS J00361617+1821104 .................. } & 2004 Dec 05 & $0.432 \times 12$ & 63.00 & $6 \times 300$ & 1.06 & \multirow[t]{2}{*}{5} \\
\hline & 2005 Oct 28 & $0.432 \times 12$ & 63.00 & $3 \times 300$ & 1.11 & \\
\hline 2MASS J22244381-0158521 _................ & 2001 Jun 15 & $0.576 \times 12$ & 63.00 & $2 \times 600$ & 1.09 & 6 \\
\hline \multirow{2}{*}{ SDSS J053951.99-005902.0 ................ } & 2001 Nov 02 & $0.432 \times 12$ & 62.78 & $8 \times 300$ & 1.30 & \multirow[t]{2}{*}{7} \\
\hline & 2005 Oct 27 & $0.432 \times 12$ & 63.00 & $3 \times 480$ & 1.15 & \\
\hline 2MASS J17281150+3948593AB ............ & 2001 Jun 15 & $0.576 \times 12$ & 63.00 & $2 \times 900$ & 1.33 & 6 \\
\hline 2MASS J16322911+1904407................. & 2001 Jun 15 & $0.576 \times 12$ & 63.00 & $2 \times 900$ & 1.37 & 8 \\
\hline DENIS-P J0255.0-4700 „.......................... & 2005 Oct 27 & $0.432 \times 12$ & 63.00 & $3 \times 420$ & 2.53 & 9 \\
\hline SDSSp J125453.90-012247.4 ................ & 2006 Jan 19 & $0.432 \times 12$ & 63.00 & $9 \times 600$ & 1.09 & 10 \\
\hline \multirow[t]{5}{*}{ 2MASS J05591914-1404488 ................. } & 2001 Nov 02 & $0.432 \times 12$ & 62.78 & $8 \times 300$ & 1.39 & \multirow[t]{5}{*}{11} \\
\hline & 2004 Dec 05 & $0.432 \times 12$ & 63.00 & $2 \times 300$ & 1.36 & \\
\hline & 2005 Oct 26 & $0.432 \times 12$ & 63.00 & $3 \times 480$ & 1.18 & \\
\hline & 2005 Oct 27 & $0.432 \times 12$ & 63.00 & $3 \times 480$ & 1.27 & \\
\hline & 2005 Oct 28 & $0.432 \times 12$ & 63.00 & $3 \times 480$ & 1.24 & \\
\hline 2MASS J15031961+2525196................. & 2006 Jan 19 & $0.432 \times 12$ & 63.00 & $2 \times 300$ & 1.07 & 12 \\
\hline SDSS J162414.37+002915.6 .................. & 2001 Jun 15 & $0.576 \times 12$ & 63.00 & $2 \times 900$ & 1.13 & 13 \\
\hline SDSS J134646.45-003150.4 „................ & 2001 Jun 15 & $0.576 \times 12$ & 63.00 & $3 \times 900$ & 1.14 & 14 \\
\hline 2MASS J15530228+1532369................ & 2001 Jun 15 & $0.576 \times 12$ & 63.00 & $2 \times 900$ & 1.04 & 15 \\
\hline 2MASS J12171110-0311131 „................ & 2001 Jun 15 & $0.576 \times 12$ & 63.00 & $2 \times 900$ & 1.15 & 16 \\
\hline 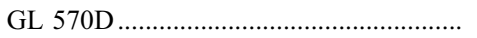 & 2001 Jun 15 & $0.576 \times 12$ & 63.00 & $2 \times 900$ & 1.45 & 17 \\
\hline 2MASS J04151954-0935066 ............... & 2005 Oct 26 & $0.432 \times 12$ & 63.00 & $2 \times 600$ & 1.18 & 15 \\
\hline
\end{tabular}

a It is also LEHPM 3396 (Pokorny et al. 2003).

b Cloudy, low transparency. Not used for $v_{\text {rot }} \sin i$ determination.

References.- (1) Stauffer et al. 1998; (2) Van Biesbroeck 1961; (3) Pokorny et al. 2003; (4) Tinney 1998; (5) Reid et al. 2000; (6) Kirkpatrick et al. 2000; (7) Fan et al. 2000; (8) Kirkpatrick et al. 1999; (9) Martín et al. 1999; (10) Leggett et al. 2000b; (11) Burgasser et al. 2000a; (12) Burgasser et al. 2003;

(13) Strauss et al. 1999; (14) Tsvetanov et al. 2000; (15) Burgasser et al. 2002b; (16) Burgasser et al. 1999; (17) Burgasser et al. 2000b.

determine the rotational velocity of brown dwarfs, with particular emphasis on the late L and T-type dwarfs, and second, study the change of rotation rate from very young ages to the ages typical of the solar neighborhood, which allows us to obtain new insight on the angular momentum evolution in the substellar regime.

\section{THE SAMPLE, OBSERVATIONS, AND DATA REDUCTION}

The sample of targets is listed in Table 1. It comprises four late $\mathrm{M}$ type dwarfs, two of which are young and lithium-bearing brown dwarfs (PP1 1 is a member of the Pleiades cluster, Stauffer et al. 1998; and LP 944-20 is a likely member of the Castor moving group, Tinney 1998; Ribas 2003). The remaining targets are six L- and nine T-type field dwarfs recently discovered by the Two Micron All Sky Survey (2MASS), Sloan, and Deep NearInfrared Survey (DENIS) surveys; discovery papers are provided in the last column of Table 1. The spectral types given in column (2) of Table 2 are taken from the literature (Kirkpatrick et al. 1995, 1997, 1999, 2000; Martín et al. 1999; Geballe et al. 2002; Burgasser et al. 2006; Phan-Bao et al. 2006). They were derived from optical and/or near-infrared low-resolution spectra and are in the interval M6.5-T8. There are objects that have been typed differently by the various groups; we provide all classifications in Table 2: first, from Kirkpatrick et al. (1999) or Burgasser et al. (2006), second, from Geballe et al. (2002), and finally, from Martín et al. (1999). For those targets with the largest discrepancies (J1632+19 and J0255-47), we adopt their mean spectral type to produce the figures of this paper. In terms of effective temperature, our sample spans the range 2700-770 K (Leggett et al. 2000a; Vrba et al. 2004). J0334-49 and vB 10 can be stars in our sample, while the remaining targets are very likely substellar.

We collected high-resolution near-infrared spectra of the 19 ultracool dwarfs using the Keck II telescope and the NIRSPEC instrument, a cross-dispersed, cryogenic echelle spectrometer employing a $1024 \times 1024$ ALADDIN InSb array detector. These observations were carried out on different occasions from 2000 December through 2006 January and are part of our large program aimed at the study of radial velocity variability. The log of the observations is shown in Table 1. In the echelle mode we selected the NIRSPEC-3 ( $J$-band) filter and an entrance slit width of 0 ".432 (i.e., 3 pixels along the dispersion direction of the detector), except for eight targets (J2224-01, J1728+39, J1632+19, J1346-00, J1624+00, J1553+15, J1217-03, and GL 570D) for which we used an entrance slit width of 0.576 . The length of both slits was $12^{\prime \prime}$. All observations were performed at an echelle angle of $\sim 63^{\circ}$. This instrumental setup provided a wavelength 
TABLE 2

Spectroscopic Rotation Velocities of Our Sample

\begin{tabular}{|c|c|c|c|c|c|}
\hline \multirow[b]{2}{*}{$\begin{array}{l}\text { Овлест } \\
\text { (1) }\end{array}$} & \multirow[b]{2}{*}{$\begin{array}{c}\text { SPECTRAL TyPE } \\
\text { (2) }\end{array}$} & \multicolumn{2}{|c|}{ Template ОвJест } & \multirow[b]{2}{*}{$\begin{array}{c}\text { Previous } \\
\left(\mathrm{km} \mathrm{s}^{-1}\right) \\
(5)\end{array}$} & \multirow[b]{2}{*}{$\begin{array}{l}\text { REFERENCE } \\
\text { (6) }\end{array}$} \\
\hline & & $\begin{array}{c}\mathrm{vB} 10 \\
\left(\mathrm{~km} \mathrm{~s}^{-1}\right) \\
(3)\end{array}$ & $\begin{array}{c}\mathrm{J} 1346-00 \\
\left(\mathrm{~km} \mathrm{~s}^{-1}\right) \\
(4)\end{array}$ & & \\
\hline PP1 1 & M6.5 & $20.7 \pm 2.4$ & $\ldots$ & $18.5 \pm 1.5$ & 1 \\
\hline vB 10 & M8 V & $\ldots$ & $\ldots$ & 6.5 & 2 \\
\hline DENIS-P J033411.39-495333.6 …..... & M9 & $\leq 15$ & $\ldots$ & & \\
\hline LP 944-20 & M9 V & $30.3 \pm 1.5$ & $\ldots$ & $30.0 \pm 2.5,28,28.3,31$ & $2,3,4,5,6$ \\
\hline 2MASS J00361617+1821104................. & $\mathrm{L} 3.5 \mathrm{~V} / \mathrm{L} 4 \mathrm{~V}$ & $36.0 \pm 2.7$ & $\ldots$ & 38,15 & 6,7 \\
\hline 2MASS J22244381-0158521 …............. & $\mathrm{L} 4.5 \mathrm{~V}$ & $30.3 \pm 3.4$ & $31.1 \pm 2.2$ & $20.9-29.2$ & 8 \\
\hline SDSS J053951.99-005902.0 ……......... & L5 V & $32.3 \pm 3.6$ & $34.0 \pm 3.4$ & & \\
\hline 2MASS J17281150+3948593AB .......... & L7 V & $25.1 \pm 7.2$ & $23.3 \pm 2.8$ & & \\
\hline 2MASS J16322911+1904407................. & L8 V/L7.5 V/dL6 & $22.6 \pm 6.7$ & $20.9 \pm 7.0$ & $30 \pm 10$ & 2,3 \\
\hline 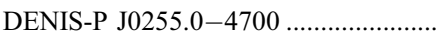 & L9 V/ /dL6 & $40.8 \pm 8.0$ & $41.1 \pm 2.8$ & $40 \pm 10$ & 2,3 \\
\hline SDSSp J125453.90-012247.4 ….......... & $\mathrm{T} 2 \mathrm{~V}$ & $29.5 \pm 5.0$ & $27.3 \pm 2.5$ & & \\
\hline 2MASS J05591914-1404488_................. & $\mathrm{T} 4.5 \mathrm{~V}$ & $25.4 \pm 5.0$ & $20.1 \pm 4.8$ & & \\
\hline 2MASS J15031961+2525196................ & $\mathrm{T} 5 \mathrm{~V}$ & $31.4 \pm 5.0$ & $32.8 \pm 2.0$ & & \\
\hline SDSS J162414.37+002915.6................. & $\mathrm{T} 6 \mathrm{~V}$ & $34.6 \pm 5.0$ & $38.5 \pm 2.0$ & & \\
\hline SDSS J134646.45-003150.4 ……........ & $\mathrm{T} 6.5 \mathrm{~V}$ & $\leq 15$ & $\ldots$ & & \\
\hline 2MASS J15530228+1532369AB .......... & $\mathrm{T} 7 \mathrm{~V}$ & $\ldots$ & $29.4 \pm 2.3$ & & \\
\hline 2MASS J12171110-0311131 ................. & $\mathrm{T} 7 \mathrm{~V} / \mathrm{T} 8 \mathrm{~V}$ & $\ldots$ & $31.4 \pm 2.1$ & & \\
\hline GL 570D & $\mathrm{T} 7.5 \mathrm{~V} / \mathrm{T} 8 \mathrm{~V}$ & $32.8 \pm 5.0$ & $28.6 \pm 2.4$ & & \\
\hline 2MASS J04151954-0935066_................ & $\mathrm{T} 8 \mathrm{~V}$ & $\ldots$ & $33.5 \pm 2.0$ & & \\
\hline
\end{tabular}

Note.-Spectroscopic rotation velocities are $v_{\text {rot }} \sin i$ measurements.

References.- - (1) Martín et al. 1998; (2) Mohanty \& Basri 2003; (3) Basri et al. 2000; (4) Reid et al. 2002; (5) Tinney \& Reid 1998; (6) Jones et al. 2005; (7) Schweitzer et al. 2001; (8) Bailer-Jones 2004.

coverage from 1.148 up to $1.346 \mu \mathrm{m}$ split into 10 different orders, a nominal dispersion ranging from 0.164 (blue wavelengths) to $0.191 \AA$ pixel $^{-1}$ (red wavelengths), and a final resolution element of $0.55-0.70 \AA$ at $1.2485 \mu \mathrm{m}$ (roughly the central wavelength of the spectra), corresponding to a resolving power $R \sim 17,800$ 22,700 . Individual exposure times were a function of the brightness of the targets, ranging from 120 to $900 \mathrm{~s}$.

Raw data were reduced using the ECHELLE package within IRAF. ${ }^{3}$ Spectra were collected at two or three different positions along the entrance slit. Nodded images were subtracted to remove sky background and dark current. White light spectra obtained with the same instrumental configuration and for each target were used for flat-fielding the data. All spectra were calibrated in wavelength using the internal arc lamp lines of $\mathrm{Ar}, \mathrm{Kr}$, and $\mathrm{Xe}$, which were typically acquired after observing the targets. The vacuum wavelengths of the arc lines were identified, and we produced fits using a third-order Legendre polynomial along the dispersion axis and a second-order one perpendicular to it. The mean rms of the fits was $0.03 \AA$, or $0.7 \mathrm{~km} \mathrm{~s}^{-1}$. We note that any systematic error or different zero-point shifts that may be present in the instrumental calibration do not affect the rotational velocity measurements. In order to correct for atmospheric telluric absorptions, near-infrared featureless stars of spectral types A0-A2 were observed several times and at different air masses during the various observing runs. The hydrogen line at $1.282 \mu \mathrm{m}$, which is intrinsic to these hot stars, was removed from the spectra before using them for division into the corresponding science data. Finally, we multiplied the science spectra by the blackbody spectrum for the temperature of $9480 \mathrm{~K}$, which is adequate for A0 V type (Allen 2000). We plotted in Figure 1 example spectra of our targets around the

\footnotetext{
${ }^{3}$ IRAF is distributed by National Optical Astronomy Observatory, which is operated by the Association of Universities for Research in Astronomy, Inc., under contract with the National Science Foundation.
}

K I doublet at $1.2485 \mu \mathrm{m}$. Effective temperature (i.e., spectral type) decreases from top to bottom. Note that all spectra have been shifted in velocity to vacuum wavelengths for a facile comparison of the atomic and molecular features. The signal-to-noise ratio of the spectra varies for different echelle orders and different

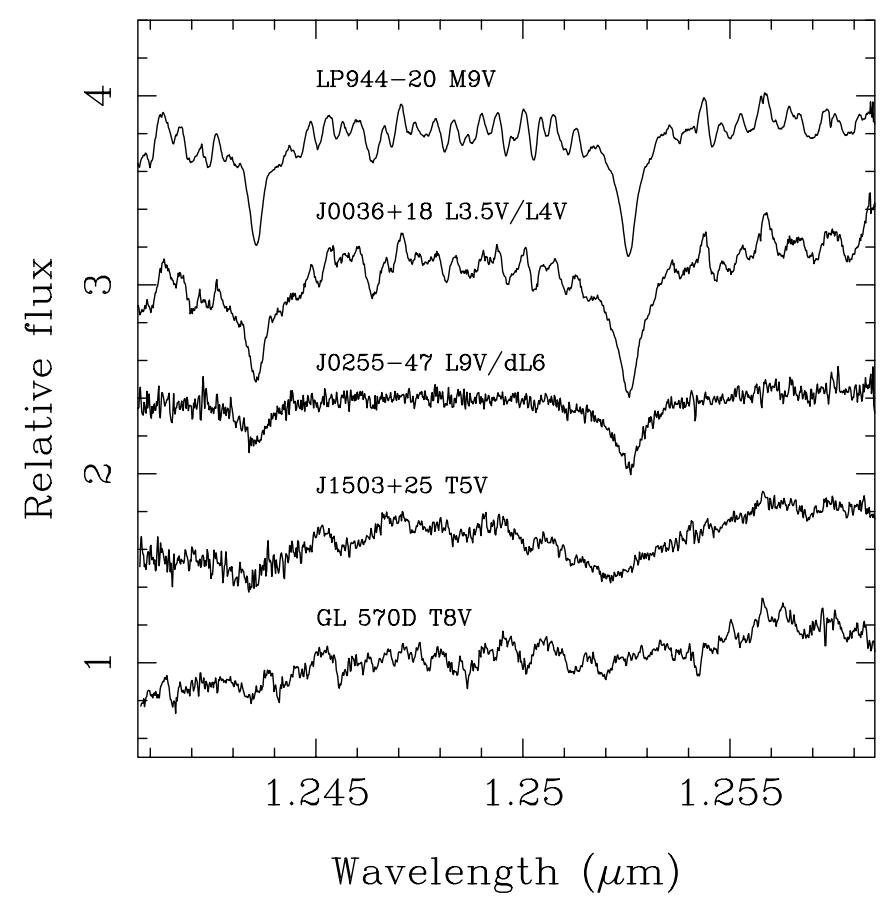

FIG. 1.-NIRSPEC spectra of some of our targets. The most prominent features of the $\mathrm{M}$ and $\mathrm{L}$ dwarfs are due to $\mathrm{K} \mathrm{I}$ absorption, which vanishes at late $\mathrm{T}$ types. Spectra are normalized to unity in the interval $1.2475-1.2490 \mu \mathrm{m}$ and offset by 0.7 on the vertical axis. All spectra are shifted in velocity to vacuum wavelengths. 
targets depending on their brightness. In general, the red orders show better signal-to-noise ratio than the blue orders, except for the reddest order centered at $1.337 \mu \mathrm{m}$ in T dwarfs, since these wavelengths are affected by strong methane and water vapor absorptions below $1300 \mathrm{~K}$.

\section{ROTATIONAL VELOCITIES}

We measured projected rotational velocities via a crosscorrelation of the spectra of our targets against spectra of slowly rotating dwarfs of similar types. The details of the procedure, which is based on the assumption that the line broadening is dominated by rotation, is fully described in the literature (e.g., Reid et al. 2002; Bailer-Jones 2004, and references therein). In summary, rotational velocities, $v_{\text {rot }} \sin i$, are obtained from the width of the peak of the cross-correlation function between the targets and the slow rotator templates. The larger the width, the faster the rotation rate. We performed the cross-correlation on each order separately, fit a Gaussian to the peak, and measured its width, which is calibrated against $v_{\text {rot }} \sin i$ by applying artificial broadening for a range of velocities $\left(10-50 \mathrm{~km} \mathrm{~s}^{-1}\right.$ in steps of $5 \mathrm{~km} \mathrm{~s}^{-1}$ ) to the spectra of slow rotators. We adopted the line rotation profile given by Gray (1992) and a limb-darkening parameter of $\epsilon=0.6$. One example of the artificially broadened spectra is displayed in Figure 2 (bottom spectrum). Only orders for which we unambiguously identified the peak of the crosscorrelation function and obtained good fits were used. Then, all results were averaged to produce the final $v_{\text {rot }} \sin i$ measurement. Uncertainties are derived from the standard deviation of the mean.

Ideally, nonrotating templates should be used. Mohanty \& Basri (2003) reported a very low projected velocity of the M8 V-type star vB $10\left(\mathrm{~km} \mathrm{~s}^{-1}\right)$. This is well below the instrumental profile broadening of our data, which is about $15 \mathrm{~km} \mathrm{~s}^{-1}$ based on 1 pixel resolution, and is also smaller than the minimum detectable rotation from our data (see below). As a result vB 10 can be considered a nonrotator in our study and has been employed as a template to obtain rotational velocities of the M- and L-type targets. The cross-correlation technique assumes that the target and template spectra are of similar type and differ only in the rotation velocity. Nevertheless, Bailer-Jones (2004) has recently shown that M-type templates can also yield accurate rotation velocities of $\mathrm{L}$ dwarfs. In our sample, the energy distribution of $\mathrm{T}$ dwarfs do differ significantly from $\mathrm{M}$ dwarfs, and we found that for the T-type targets the cross-correlation with $\mathrm{vB} 10$ gives reasonable results if resonance lines of $\mathrm{K}$ I are avoided and only molecular (particularly water vapor) lines are used in the cross-correlation. The cool brown dwarf J1346-00 (T6.5 V) turns out to have a low value of $v_{\text {rot }} \sin i$. (Because of the unknown inclination angle of the rotation axis, we cannot establish whether J1346-00 is a true fast or slow rotator. However, we used it as a "slow rotator" in our study.) From the data we can impose an upper limit of $15 \mathrm{~km} \mathrm{~s}^{-1}$ on its projected rotational velocity. We used $\mathrm{J} 1346-00$ as a secondary template to measure rotational broadening of the coolest objects in our sample, the late L and T-type dwarfs. We note, however, that using a rotating template will underestimate the rotation velocities (Mohanty \& Basri [2003] provide an extensive and quantitative discussion on this respect). Our derived $v_{\text {rot }} \sin i$ measurements using vB 10 and J1346-00 as the slowly rotating reference objects are given in columns (3) and (4) of Table 2, respectively. The agreement between the two columns is good within $1 \sigma$ (i.e., there is no obvious tendency for $v_{\text {rot }} \sin i$ obtained using J1346-00 to be lower than the values derived with the M-type dwarf). For those objects in common with other studies (two M dwarfs and four L dwarfs), we also give their previously derived projected rotational velocities

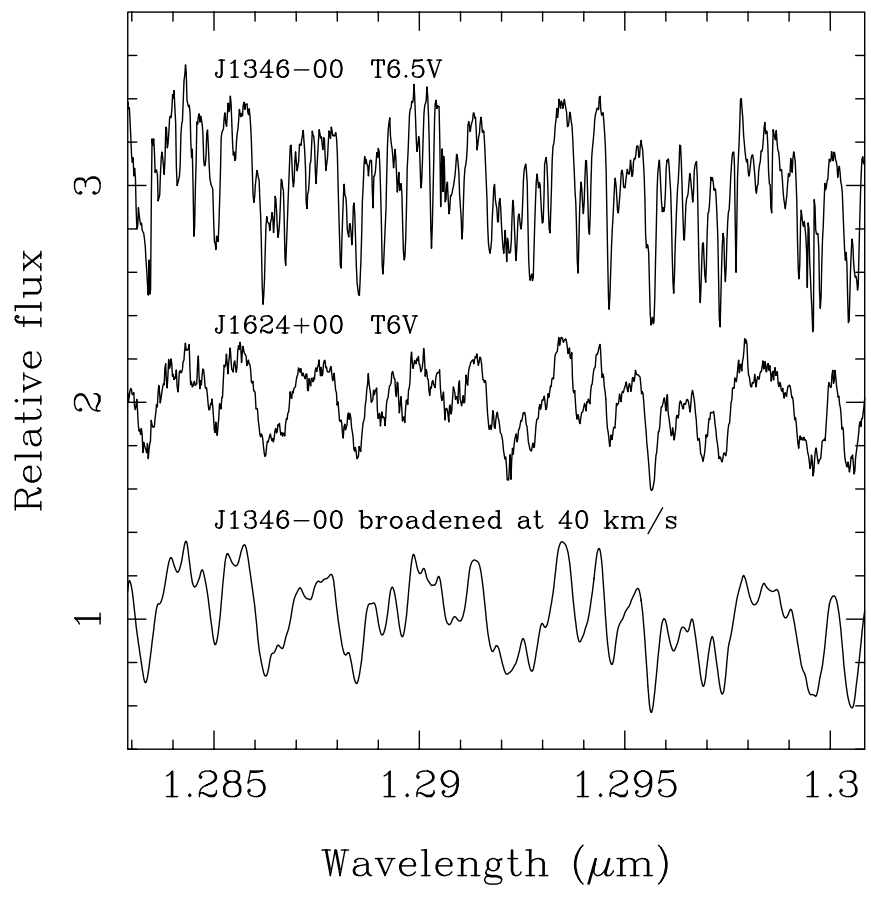

FIG. 2.-NIRSPEC spectra of the brown dwarf J1346-00 (T6.5 V, low $v_{\text {rot }} \sin i$; top line) and the rapid rotator $\mathrm{J} 1624+00(\mathrm{~T} 6 \mathrm{~V}$; middle). The spectrum of $\mathrm{J} 1624+00$ has been shifted in velocity to match $\mathrm{J} 1346-00$ for a facile comparison of features. The spectrum of J1346-00 convolved with a rotation profile of $40 \mathrm{~km} \mathrm{~s}^{-1}$ is shown at the bottom. Data are scaled to a common vertical range and offset by unity on the vertical axis for clarity.

in column (5) of Table 2 . With the only exception of J0036+18 (see below), our measurements and the values from the literature agree within the expected errors.

Figure 2 depicts the spectra of the T-type secondary template $(\mathrm{J} 1346-00)$ and of one rapid rotator of similar classification (J1624+00, T6 V). The data of J1624+00 were shifted in velocity to match the template. For comparison purposes the spectrum of $\mathrm{J} 1346-00$ broadened at the (roughly) rotation velocity of J1624+ 00 is also shown at the bottom of the figure. The resemblance of $\mathrm{J} 1624+00$ to the artificially broadened spectrum is remarkable, supporting the $v_{\text {rot }} \sin i$ results of the cross-correlation technique.

In our sample of 19 ultracool dwarfs, only three of them have small values of $v_{\text {rot }} \sin i$, the reference M8-type dwarf vB 10 , the nearby M9 dwarf J0334-49, and the T6.5-type object J1346-00. For the latter two we have placed an upper limit of $15 \mathrm{~km} \mathrm{~s}^{-1}$. The cross-correlation technique suggests that the M9-dwarf rotates at a speed of $8.5 \pm 2.6 \mathrm{~km} \mathrm{~s}^{-1}$. However, this measurement is below the minimum detectable projected rotational velocity of our study, which we estimate at about $10.5 \mathrm{~km} \mathrm{~s}^{-1}$ after the discussion by Bailer-Jones (2004). Therefore, the adoption of the mean NIRSPEC instrumental broadening as the upper limit on the $v_{\text {rot }} \sin i$ for the slowest rotators in our sample is conservative. It may be noteworthy that we have found a slowly rotating $\mathrm{T}$ dwarf in a sample of nine T-type dwarfs, while a much larger sample of L dwarfs drawn from our work and the literature has yet to yield one. However, we note that there are a few L dwarfs with $v_{\text {rot }} \sin i \sim 10 \mathrm{~km} \mathrm{~s}^{-1}$ and that the rotation inclinations are randomly distributed.

\section{DISCUSSION}

\subsection{Periodic Photometric Variability and $v_{\mathrm{rot}} \sin i$}

Four L-type dwarfs among our targets have tentative rotational periods available in the literature. Berger et al. (2005) reported on 
the detection of strongly variable and periodic radio emission in $\mathrm{J} 0036+18(\mathrm{~L} 3.5 \mathrm{~V})$ with a periodicity of $3 \mathrm{hr}$. These authors suggested various scenarios to account for the observed periodic radio emission, one of which is related to equatorial rotation and an anchored, long-lived magnetic field. The period of $3 \mathrm{hr}$ is consistent with our $v_{\text {rot }} \sin i$ measurement $\left(36.0 \pm 2.7 \mathrm{~km} \mathrm{~s}^{-1}\right)$; thus, it is plausibly the true rotation periodicity. Using a radius of $0.09 R_{\odot}$, the inferred inclination angle of the rotation axis is $i \geq 66^{\circ}$ with an expectation value of $81^{\circ}$. We note, however, that our $v_{\text {rot }} \sin i$ differs by a factor of 2.4 with respect to the measurement given by Schweitzer et al. (2001). These authors employed a different technique to determine rotation velocities; they compared artificially broadened theoretical spectra to observed spectra. Using a similar approach, Jones et al. (2005) obtained a rotation velocity (see Table 2) in better agreement with our measurement.

Recently, Koen (2005) has discussed the possible variable nature of J0255-47 (L9 V/dL6) on more than one timescale. This author found two possible photometric modulations with periodicities of 1.7 and $5.2 \mathrm{hr}$ in the optical light curve of J0255-47. If this object has a radius of $0.09 R_{\odot}$, the longest period may be ruled out as the rotation period. However, the shortest periodicity is consistent with a rotational modulation, and when combined with our $v_{\text {rot }} \sin i$ it would yield a rotation axis inclination angle of $i \sim 40^{\circ}$. Then, the true equatorial velocity of J0255-47 would turn out to be $\sim 64 \mathrm{~km} \mathrm{~s}^{-1}$.

The L5 V dwarf J0539-00 has also been detected to be photometrically variable by Bailer-Jones \& Mundt (2001), with a derived periodicity of $13.3 \mathrm{hr}$. In addition, a tentative modulation of $21.8 \mathrm{hr}$ associated to the L4.5 V dwarf J2224-01 has been reported by Gelino et al. (2002). Given our $v_{\text {rot }} \sin i$ data and the radius of $0.09 R_{\odot}$, such long periods cannot be securely ascribed to rotation modulation of a long-lived atmospheric feature. Similarly, other ultracool dwarfs show periodic variability that is too long to be related to the rotation period (e.g., Martín et al. 2001; Gelino et al. 2002). Nevertheless, long periodicities could be interpreted as due to equatorial rotation if these objects had larger size, i.e., were significantly younger than the rest of the objects in the solar neighborhood.

\subsection{Rotation and Angular Momentum Evolution}

From Table 2 field T-type brown dwarfs are relatively fast rotators with $v_{\text {rot }} \sin i$ measurements in the interval $\leq 15-40 \mathrm{~km} \mathrm{~s}^{-1}$. All state-of-the-art evolutionary models available in the literature predict that the size of brown dwarfs keeps approximately constant $\left(0.08-0.1 R_{\odot}\right)$ at ages older than $\sim 0.5 \mathrm{Gyr}$, regardless of the mass. Under the assumption that this theoretical prediction is correct, the rotation periods of T-type brown dwarfs are probably below $12.5 \mathrm{hr}$. To date only a few T dwarfs have been photometrically monitored to search for rotational modulation (e.g., Burgasser et al. 2002a; Goldman 2003; Koen 2005). Enoch et al. (2003) found evidence for periodic behavior on a timescale of $3 \mathrm{hr}$ in one T1 brown dwarf. Unfortunately, this variable object is not in our sample; nevertheless, the derived photometric periodicity is consistent with our prediction for T-type brown dwarfs based on the radii given by the evolutionary models.

Our $v_{\mathrm{rot}} \sin i$ results are plotted against spectral type in Figure 3, where we have also included data of field M stars and L dwarfs from the literature (Marcy \& Chen 1992; Basri \& Marcy 1995; Delfosse et al. 1998; Basri et al. 2000; Reid et al. 2002; Mohanty \& Basri 2003; Bailer-Jones 2004). The $v_{\text {rot }} \sin i$ measurement of the unresolved T-dwarf binary $\epsilon$ Ind Bab obtained by Smith et al. (2003) is also plotted in the figure. Note that many early to mid-M stars have very slow rotation $\left(v_{\mathrm{rot}} \sin i \leq 5 \mathrm{~km} \mathrm{~s}^{-1}\right.$; Delfosse et al. 1998; Mohanty \& Basri 2003), and for the sake

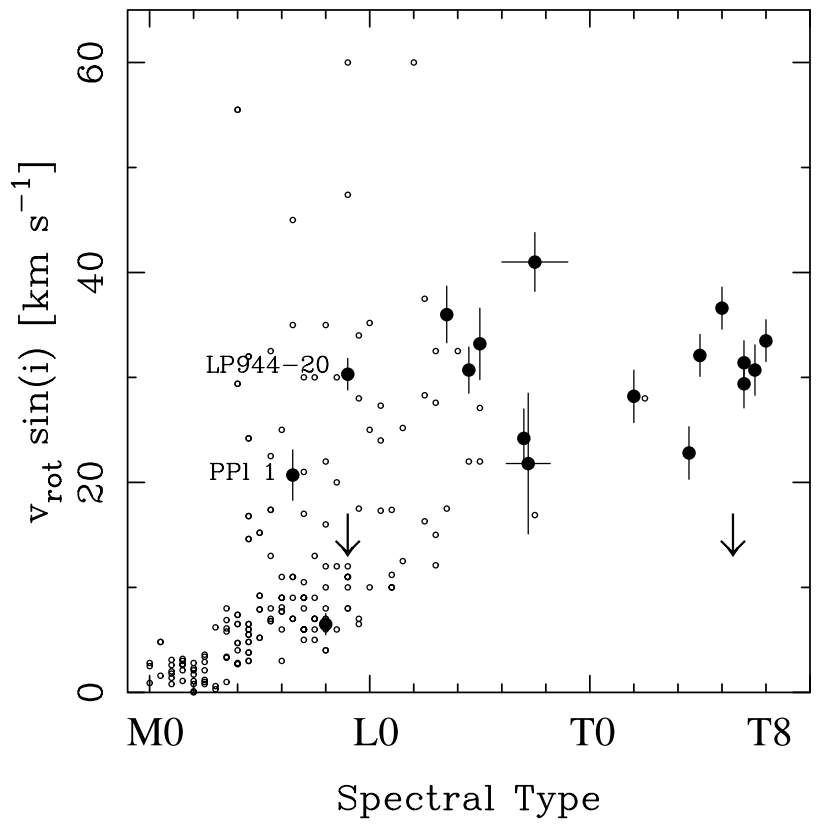

FIG. 3.- Spectroscopic rotation velocities of M-, L- and T-type dwarfs. Our sample is plotted as filled circles and arrows denoting positive detections and upper limits, respectively. The two known young dwarfs of our sample are labeled. The datum of vB 10 (M8 V), one of the template sources for rotation velocity measurement, is taken from Mohanty \& Basri (2003). Two L dwarfs show large error bars in spectral type to account for their different classifications found in the literature. Small open circles correspond to data (positive detections) from the literature (see text). We note that a large fraction of M0-M4 stars have nondetections $\left(v_{\mathrm{rot}} \sin i \leq 5 \mathrm{~km} \mathrm{~s}^{-1}\right)$, and their upper limits are not plotted here for clarity.

of clarity we have not plotted in the figure the upper limits on their detection. It is obvious that there is a correlation between rotation and spectral type; rotational velocity keeps increasing with later spectral type from $\mathrm{M}$ to $\mathrm{T}$ types. This behavior was previously reported for $\mathrm{M}$ and $\mathrm{L}$ dwarfs in the literature (Mohanty \& Basri 2003; Bailer-Jones 2004) and is in part due to the different mass of the objects contemplated in Figure 3. We do not consider the young brown dwarfs of our sample, PP1 1 and LP 944-20, in the following discussion. At the typical age interval of the solar vicinity $(\sim 1-10 \mathrm{Gyr})$, T-type objects are substellar with likely masses in the brown dwarf regime (20-70 $M_{\text {Jup }}$; Baraffe et al. 2003). On the contrary and for the same age interval, low-mass and very low mass field stars above the substellar limit have $\mathrm{M}$ and early L types. From Figure 3 and despite the uncertainty introduced by the inclination angle of the rotation axis, field brown dwarfs indeed rotate more rapidly than field M-type stars. Scholz \& Eislöffel $(2004,2005)$ studied the rotation period-mass relationship of stellar and substellar members in the $\sigma$ Orionis and $\epsilon$ Orionis young clusters, finding that the lower the mass, the shorter the rotation period.

Field L-type dwarfs can be either very low mass stars with probable masses below $0.1 M_{\odot}$ or brown dwarfs depending on age and surface temperature. About $35 \%$ of L3 V-L4 V dwarfs have lithium in their spectra, and the rate increases up to $70 \%$ at L6 V (Kirkpatrick et al. 2000). These are definitively substellar with likely masses below $60 M_{\text {Jup }}$. L-type objects are also reported in the literature to be fast rotators (Basri et al. 2000; Bailer-Jones 2004), with projected rotational velocities up to $60 \mathrm{~km} \mathrm{~s}^{-1}$ and a minimum velocity of $10 \mathrm{~km} \mathrm{~s}^{-1}$ (Mohanty \& Basri 2003). From our data T-type brown dwarfs do not appear to have significantly faster rotation than $\mathrm{L}$ dwarfs, and a smooth transition between the L- and T-types can be seen in Figure 3 . 


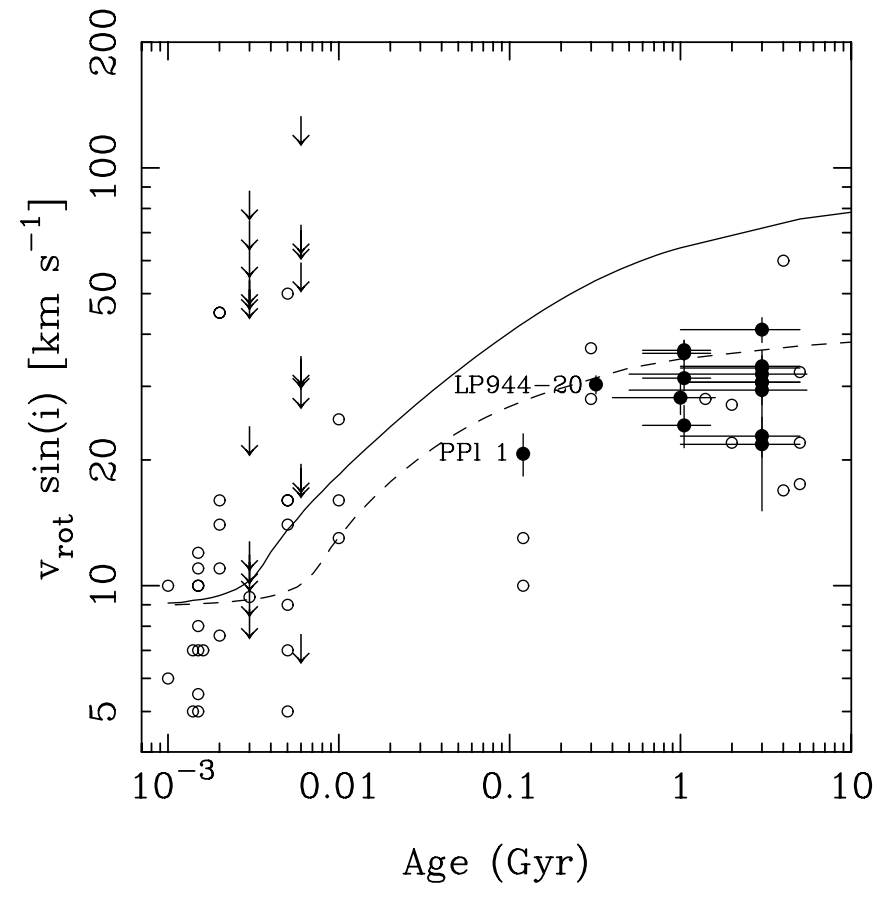

FIG. 4.-Projected rotation velocity as a function of age for brown dwarfs with likely masses in the interval 30-70 $M_{\text {Jup }}$. Our data (PP1 1, LP 944-20, and field dwarfs later than L3.5 V) are plotted as filled circles. Data from the literature (see text for details) are shown with open circles and arrows (upper limits). The curves trace the hydrostatic contraction of brown dwarfs with $60 M_{\text {Jup }}$ (solid line) and $30 M_{\text {Jup }}$ (dashed line), which have a rotation velocity of $9 \mathrm{~km} \mathrm{~s}^{-1}$ at the age of $1 \mathrm{Myr}$.

However, we note that there is a hint for the lower envelope of the $v_{\text {rot }} \sin i$-spectral-type relation to increase linearly from mid-M to $\mathrm{T}$ types. This trend may be an artifact of the small sample size of $\mathrm{T}$ dwarfs and of our detection limit; hence, more measurements of different T-type brown dwarfs would be required to definitively ascertain this tendency.

Also relevant is the exploration of the angular momentum evolution of brown dwarfs. To address this issue we should know the age of our sample. PPl 1 is a Pleiades brown dwarf (60-72 $\left.M_{\text {Jup }}\right)$ with an estimated age of $120 \mathrm{Myr}$. We have calculated the space motions of all the other targets except for two T dwarfs that lack parallax measurements (forthcoming paper) and used the criteria defined by Leggett (1992) to group them into young disk, youngold disk, and old disk kinematic categories. LP 944-20 was widely discussed by Ribas (2003). According to their Galactic velocities, all $\mathrm{L}$ and $\mathrm{T}$ dwarfs of Table 2 can be classified as members of the young and young-old disk, for which we have assumed the age intervals $0.6-1.5 \mathrm{Gyr}$ (young disk) and $1-5 \mathrm{Gyr}$ (young-old disk). We note that Reid et al. (2002) also pointed out that late $M$ dwarfs appear kinematically younger than early $M$ stars and that young $\mathrm{M}$ dwarfs rotate faster than the older ones. From our data we find no difference in $v_{\text {rot }} \sin i$ between the young disk $\mathrm{T}$ dwarfs and the young-old disk ones.

Our $v_{\text {rot }} \sin i$ values are plotted against age in Figure 4, where we have considered only brown dwarfs ( PPl 1, LP 944-20, and the field dwarfs cooler than L3.5 V). Further spectroscopic rotation velocities of confirmed lithium-bearing Pleiades brown dwarfs and of the substellar pair GJ 569Ba and GJ 569Bb are obtained from the literature (Basri \& Marcy 1995; Martín et al. 1998; Zapatero Osorio et al. 2004) and are also shown in the figure. Despite the scarce number of data for ages of hundreds of megayears, there is no clear evidence for a significant braking between the Pleiades age and the field. This contrasts markedly to solar-type stars. At the age of the Hyades ( $600 \mathrm{Myr})$, most
G- and K-type stars are rather slow rotators, and some M-type stars display relatively rapid rotation with maximum $v_{\text {rot }} \sin i=$ $20 \mathrm{~km} \mathrm{~s}^{-1}$ (Stauffer et al. 1997).

We have compiled the $v_{\text {rot }} \sin i$ measurements of very young brown dwarfs with ages between $\sim 1$ and 10 Myr from Joergens \& Guenther (2001), Muzerolle et al. (2003), and Mohanty et al. (2005). These data correspond to confirmed substellar members of the following star-forming regions and young clusters: $\rho$ Ophiuchi ( $1 \mathrm{Myr})$, Taurus ( 1.5 Myr), IC 348 ( 2 Myr), Chamaeleon I ( $\sim 2$ Myr), $\sigma$ Ori ( $\sim 3$ Myr), Upper Scorpius $(\sim 5 \mathrm{Myr})$, and TW Hya ( $\sim 10 \mathrm{Myr})$. It is accepted that the substellar limit is located at about M6-M6.5 spectral type at these young ages, as well as in the Pleiades (e.g., Luhman et al. 1998; Martín et al. 1998). Hence, to secure that only brown dwarfs are depicted in Figure 4, we have plotted sources with spectral type M7 and later (the coolest brown dwarf of these spectroscopic studies has spectral class M9.5). Their masses are likely in the interval 30-70 $M_{\text {Jup }}$, i.e., fully overlapping with the mass estimates for the field T-type objects of our sample. The projected rotation velocities of the very young $(\leq 10 \mathrm{Myr})$ brown dwarfs span the range $0-60 \mathrm{~km} \mathrm{~s}^{-1}$. In addition, several groups have photometrically monitored substellar members of the $\sigma$ Ori and $\epsilon$ Ori ( 6 Myr) clusters (Bailer-Jones \& Mundt 2001; Scholz \& Eislöffel 2004, 2005; Zapatero Osorio et al. 2003; Caballero et al. 2004), finding periodic behaviors on timescales between 3 and $80 \mathrm{hr}$. The various groups of authors discuss that the observed modulated variability is possibly related to rotation in many cases. We have used the radii predictions of Baraffe et al. (2003) to transform periods into velocities, which are plotted as arrows (denoting upper limits) in Figure 4. From the figure it is noteworthy that a significant amount $(\sim 50 \%)$ of young, massive brown dwarfs have $v_{\text {rot }} \sin i \leq 10 \mathrm{~km} \mathrm{~s}^{-1}$, while in general, field substellar objects of similar mass rotate 3-6 times faster. Brown dwarfs definitively undergo a spin-up because of gravitational contraction, and their spin-down time appears to be longer than that of solar-type stars. Chabrier \& Küker (2006) argued that the magnetic field topology of fully convective objects exhibits an asymmetric structure, which in addition to the decreasing conductivity in the atmosphere of ultracool dwarfs reduces the efficiency of magnetic braking, providing a possible explanation for the lower angular momentum loss of brown dwarfs.

Nevertheless, fast-rotating brown dwarfs do exist at all ages from a few megayears through several gigayears. This is independent of evolutionary models, since rotation periods of a few hours and $v_{\text {rot }} \sin i \geq 10 \mathrm{~km} \mathrm{~s}^{-1}$ are equally observed among young and field brown dwarfs. This suggests some angular momentum loss throughout the lifetime of a brown dwarf. Furthermore, with the current evolutionary models, simple hydrostatic contraction (i.e., angular momentum conservation) fails to reconcile the entire $v_{\text {rot }} \sin i$ distributions of the young ( $\leq 10 \mathrm{Myr}$ ) and "old" ( $\geq 100 \mathrm{Myr}$ ) substellar populations shown in Figure 4. As an example we have plotted in the $v_{\text {rot }} \sin i$-age diagram of Figure 4 the purely hydrostatic contraction of two presumed brown dwarfs with masses of 30 (dashed line) and $60 M_{\text {Jup }}$ (solid line) by assuming a moderate "initial" rotation velocity of $9 \mathrm{~km} \mathrm{~s}^{-1}$ and the theoretical models of Baraffe et al. (2003). We note that $60 M_{\text {Jup }}$ is probably the most representative mass of the objects in Figure 4 . At the very young ages there are brown dwarfs located above and below the curves of angular momentum conservation; however, for ages older than $1 \mathrm{Gyr}$, nearly all brown dwarfs lie below the curves. Hence, some braking becomes apparent. We remark that the conclusions drawn from this discussion are based on current evolutionary models and should be revised if the models are proved to be wrong. 
The fastest rotating young brown dwarfs with masses in the interval 50-72 $M_{\text {Jup }}$, periods below $10 \mathrm{hr}$, and $v_{\text {rot }} \geq 45 \mathrm{~km} \mathrm{~s}^{-1}$ (note the drop of $\sin i$ ) pose a problem to theory (Scholz et al. 2005). Relying on actual models, these rapid, massive substellar rotators would reach the spherical breakup velocity in less than the lifetime of the Galaxy unless they brake down dramatically. It is expected that fast rotation impacts the evolution of brown dwarfs. These objects have rather neutral atmospheres (low conductivity) and despite the fact they can hold some long-lived magnetic fields up to a few gigayears (Berger et al. 2005, and references therein), the atmospheric fluid motions and the field are likely decoupled, which reduces the "magnetic" activity. The mechanisms through which ultracool (particularly L and T type), fully convective rotators can lose angular momentum are to be determined (magnetic braking, frictional heating, etc.). However, it is worth mentioning that young massive brown dwarfs of mid to late $\mathrm{M}$ spectral classes are warm enough to have coronae in a manner similar to low-mass stars (see, e.g., Preibisch \& Zinnecker 2002; Mokler \& Stelzer 2002; Bouy 2004; Preibisch et al. 2005, and references therein). This could in principle provide a braking mechanism, but at a time when the brown dwarfs have not yet fully contracted and have not cooled down significantly. Because there is no obvious braking between the Pleiades and field brown dwarfs (Fig. 4), it might be possible that these objects spin up due to contraction and brake down while still being warm (and very young), and once they evolve to cooler temperatures, the braking time becomes longer. Very recently, Reiners \& Basri (2006) report on the high $v_{\text {rot }} \sin i$ of an halo L dwarf, suggesting that braking is even inexistent (provided the object has an "halo" age). In addition, flare activity, like the one reported in a few field $\mathrm{L}$ dwarfs (e.g., Liebert et al. 2003) can also shed off angular momentum. Nevertheless, the frequency of strong flares in cool objects is quite low ( $\leq 7 \%$; Gizis et al. 2000), so their contribution to the rotation braking is expected to be small. Finally, we point out that there can be a diversity of angular momentum histories in our sample of brown dwarfs; some could be binaries, some may have flares, etc.

\section{SUMMARY AND CONCLUSIONS}

We obtained high-resolution $(R \sim 17,800-22,700)$, nearinfrared $(1.148-1.346 \mu \mathrm{m})$ spectra of a sample of 19 ultracool dwarfs (M6.5-T8) using the NIRSPEC instrument and the $10 \mathrm{~m}$ Keck II telescope. Our sample comprises two late M dwarfs, two young (100-500 Myr) brown dwarfs, and 15 L3.5-T8 likely brown dwarfs of the solar neighborhood. Projected rotation velocities $\left(v_{\text {rot }} \sin i\right)$ were measured via a cross-correlation of the data against spectra of slow-rotating dwarf templates of similar types, which were observed with the same instrumental configuration. The star vB $10(\mathrm{M} 8 \mathrm{~V})$ and the brown dwarf J1346-00 (T6.5 V) were the templates. We found that in general the T-type brown dwarfs are relatively fast rotators, as are the $\mathrm{L}$ dwarfs, with $v_{\text {rot }} \sin i$ spanning the range $\leq 15-40 \mathrm{~km} \mathrm{~s}^{-1}$. Assuming a radius of $0.08-0.1 R_{\odot}$, we placed an upper limit of $12.5 \mathrm{hr}$ on the equatorial rotation of $\mathrm{T}$ dwarfs. Rotation appears to correlate with spectral type (from $\mathrm{M}$ to T class), $v_{\text {rot }} \sin i$ increasing toward later types. However, we found no clear evidence for $\mathrm{T}$ dwarfs rotating significantly faster than L dwarfs.

We compared our $v_{\text {rot }} \sin i$ measurements to photometric periods of four $\mathrm{L}$ dwarfs reported in the literature and concluded that the $3 \mathrm{hr}$ periodic radio emission of J0036+18 (Berger et al. 2005 ) is likely the true rotation period of the object. However, the derived photometric periodicities of J0255-47 (5.2 hr; Koen 2005), J0539-00 (13.3 hr; Bailer-Jones \& Mundt 2001), and J2224-01 (21.8 hr; Gelino et al. 2002) seem too long to be considered the rotation periods if we assume a radius of $0.09 R_{\odot}$.

We also compared our data to $v_{\text {rot }} \sin i$ measurements of brown dwarfs of similar mass (30-72 $M_{\text {Jup }}$ ), which are confirmed members of very young star-forming regions and star clusters (1-10 Myr). A significant fraction of the very young brown dwarfs show $v_{\text {rot }} \sin i \leq 10 \mathrm{~km} \mathrm{~s}^{-1}$, which contrasts with the high values found in field brown dwarfs. This supports the fact that brown dwarfs spin up with age due to gravitational contraction. From the calculation of the hydrostatic contraction based on radii predictions of state-of-the-art evolutionary models, we conclude that brown dwarfs seem to brake down from 1 Myr to the age of the solar neighborhood, and hence, they lose some angular momentum. However, massive brown dwarfs do not appear to brake down as dramatically as solar-type to early $\mathrm{M}$ stars, suggesting that their spin-down time is significantly longer.

We thank V. J. S. Béjar for useful discussions. We also thank G. Basri (the referee of this paper) for his comments and suggestions. This research has been supported by a Keck PI Data Analysis grant awarded to E. L. M. by Michelson Science Center, and by the Spanish projects AYA2003-05355 and AYA200506453. We thank the Keck observing assistants and the staff in Waimea for their kind support. The data were obtained at the W. M. Keck Observatory, which is operated as a scientific partnership between the California Institute of Technology, the University of California, and NASA. The Observatory was made possible by the generous financial support of the W. M. Keck Foundation. The authors extend special thanks to those of Hawaiian ancestry, on whose sacred mountain we are privileged to be guests. This research has made use of the SIMBAD database, operated at CDS, Strasbourg, France.

\section{REFERENCES}

Allen, W. B. 2000, in Allen's Astrophysical Quantities, ed. A. N. Cox (4th ed; New York: Springer), 151

Bailer-Jones, C. A. L. 2004, A\&A, 419, 703

Bailer-Jones, C. A. L., \& Mundt, R. 2001, A\&A, 367, 218

Baraffe, I., Chabrier, G., Barman, T. S., Allard, F., \& Hauschildt, P. 2003, A\&A, 402, 701

Basri, G., \& Marcy, G. W. 1995, AJ, 109, 762

Basri, G., Mohanty, S., Allard, F., Hauschildt, P. H., Delfosse, X., Martín, E. L., Forveille, T., \& Goldman, B. 2000, ApJ, 538, 363

Berger, E., et al. 2005, ApJ, 627, 960

Bouy, H. 2004, A\&A, 424, 619

Burgasser, A. J., Geballe, T. R., Leggett, S. K., Kirkpatrick, J. D., \& Golimowski, D. A. 2006, ApJ, 637, 1067

Burgasser, A. J., Kirkpatrick, J. D., McElwain, M. W., Cutri, R. M., Burgasser, A. J., \& Skrutskie, M. F. 2003, AJ, 125, 850

Burgasser, A. J., Liebert, J., Kirkpatrick, J. D., \& Gizis, J. E. 2002a, AJ, 123, 2744

Burgasser, A. J., et al. 1999, ApJ, 522, L65

2000a, AJ, 120, 1100

. 2000b, ApJ, 531, L57

. 2002b, ApJ, 564, 421

Burrows, A., et al. 1997, ApJ, 491, 856

Caballero, J. A., Béjar, V. J. S., Rebolo, R., \& Zapatero Osorio, M. R. 2004, A\&A, 424, 857

Chabrier, G., \& Küker, M. 2006, A\&A, 446, 1027

Dahn, C. C., et al. 2002, AJ, 124, 1170

Delfosse, X., Forveille, T., Perrier, C., \& Mayor, M. 1998, A\&A, 331, 581

Enoch, M. L., Brown, M. E., \& Burgasser, A. J. 2003, AJ, 126, 1006

Fan, X., et al. 2000, AJ, 119, 928

Geballe, T. R., et al. 2002, ApJ, 564, 466 
Gelino, C. R., Marley, M. S., Holtzman, J. A., Ackerman, A. S., \& Lodders, K. 2002, ApJ, 577, 433

Gizis, J. E., Monet, D. G., Reid, I. N., Kirkpatrick, J. D., Liebert, J., \& Williams, R. J. 2000, AJ, 120, 1085

Goldman, B. 2003, in IAU Symp. 211, Brown Dwarfs, ed. E. L. Martín (San Francisco: ASP), 461

Gray, D. F. 1992, The Observation and Analysis of Stellar Photospheres (2nd ed.; Cambridge: Cambridge Univ. Press)

Joergens, V., \& Guenther, E. 2001, A\&A, 379, L9

Jones, H. R. A., Pavlenko, Ya., Viti, S., Barber, R. J., Yakovina, L. A., Pinfield, D., \& Tennyson, J. 2005, MNRAS, 358, 105

Kirkpatrick, J. D., Henry, T. J., \& Irwin, M. J. 1997, AJ, 113, 1421

Kirkpatrick, J. D., Henry, T. J., \& Simons, D. A. 1995, AJ, 109, 797

Kirkpatrick, J. D., et al. 1999, ApJ, 519, 802 2000, AJ, 120, 447

Koen, C. 2005, MNRAS, 360, 1132

Leggett, S. K. 1992, ApJS, 82, 351

Leggett, S. K., Allard, F., Dahn, C., Hauschildt, P. H., Kerr, T. H., \& Rayner, J. 2000a, ApJ, 535, 965

Leggett, S. K., et al. 2000b, ApJ, 536, L35

Liebert, J., Kirkpatrick, J. D., Cruz, K. L., Reid, I. N., Burgasser, A., Tinney, C. G., \& Gizis, J. E. 2003, AJ, 125, 343

Luhman, K. L., Briceño, C., Rieke, G. H., \& Hartmann, L. 1998, ApJ, 493, 909

Marcy, G. W., \& Chen, G. H. 1992, ApJ, 390, 550

Martín, E. L., Basri, G., Gallegos, J. E., Rebolo, R., Zapatero Osorio, M. R., \& Béjar, V. J. S. 1998, ApJ, 499, L61

Martín, E. L., Delfosse, X., Basri, G., Goldman, B., Forveille, T., \& Zapatero Osorio, M. R. 1999, AJ, 118, 2466

Martín, E. L., Zapatero Osorio, M. R., \& Lehto, H. 2001, ApJ, 557, 822

Mohanty, S., \& Basri, G. 2003, ApJ, 583, 451

Mohanty, S., Jayawardhana, R., \& Basri, G. 2005, ApJ, 626, 498

Mokler, F., \& Stelzer, B. 2002, A\&A, 391, 1025
Muzerolle, J., Hillenbrand, L., Calvet, N., Briceño, C., \& Hartmann, L. 2003, ApJ, 592, 266

Nakajima, T., Tsuji, T., \& Yanagisawa, K. 2004, ApJ, 607, 499

Phan-Bao, N., et al. 2006, MNRAS, 366, L40

Pokorny, R. S., Jones, H. R. A., \& Hambly, N. C. 2003, A\&A, 397, 575

Preibisch, T., \& Zinnecker, H. 2002, AJ, 123, 1613

Preibisch, T., et al. 2005, ApJS, 160, 582

Reid, I. N., Kirkpatrick, J. D., Gizis, J. E., Dahn, C. C., Monet, D. G., Williams,

R. J., Liebert, J., \& Burgasser, A. J. 2000, AJ, 119, 369

Reid, I. N., Kirkpatrick, J. D., Liebert, J., Gizis, J. E., Dahn, C. C., \& Monet, D. G. 2002, AJ, 124, 519

Reiners, A., \& Basri, G. 2006, AJ, 131, 1806

Ribas, I. 2003, A\&A, 400, 297

Scholz, A., \& Eislöffel, J. 2004, A\&A, 419, 249 2005, A\&A, 429, 1007

Scholz, A., Jayawardhana, R., Eislöffel, J., \& Froebrich, D. 2005, Astron. Nachr., 326, 895

Schweitzer, A., Gizis, J. E., Hauschildt, P. H., Allard, F., \& Reid, I. N. 2001, ApJ, 555, 368

Smith, V. et al. 2003, ApJ, 599, L107

Stauffer, J. R., Balachandran, S. C., Krishnamurthi, A., Pinsonneault, M., Terndrup,

D. M., \& Stern, R. A. 1997, ApJ, 475, 604

Stauffer, J. R., Schultz, G., \& Kirkpatrick, J. D. 1998, ApJ, 499, L199

Strauss, M. A., et al. 1999, ApJ, 522, L61

Tinney, C. G. 1998, MNRAS, 296, L42

Tinney, C. G., \& Reid, I. N. 1998, MNRAS, 301, 1031

Tsvetanov, Z. I., et al. 2000, ApJ, 531, L61

Van Biesbroeck, G. 1961, AJ, 66, 528

Vrba, F. J., et al. 2004, AJ, 127, 2948

Zapatero Osorio, M. R., Caballero, J. A., Béjar, V. J. S., \& Rebolo, R. 2003, A\&A, 408, 663

Zapatero Osorio, M. R., Lane, B. R., Pavlenko, Ya., Martín, E. L., Britton, M., \& Kulkarni, S. R. 2004, ApJ, 615, 958 